\title{
DESCRIPCIÓN DEL DOPING EN EL EQUINO Y EVALUACIÓN EN CHILE.
}

\author{
Christian Tuemmers ${ }^{*} \&$ Angélica Saldivia \\ Universidad Católica de Temuco, Facultad de Recursos Naturales, Escuela de Medicina Veterinaria, Casilla \\ 15-D, Temuco, Chile.
}

*Autor para correspondencia: ctuemmers@uct.cl

Resumen.

El doping consiste en cualquier método, ya sea físico o químico que altere el rendimiento y la capacidad competitiva de un equino. A través de los años han existido muchas culturas que han utilizado al equino como una herramienta de trabajo por ende la necesidad de aumentar el rendimiento de los animales data de tiempos tan antiguos que aún se desconoce el verdadero inicio de esta técnica. Hoy en día el uso de sustancias que alteren el rendimiento del equino en un evento deportivo está estrictamente prohibido, aun así, esta prohibición queda en mano de las organizaciones involucradas en cada país.

La federación ecuestre internacional ha establecido la existencia de sustancias que no deberían estar en el organismo de un caballo en ningún momento, denominadas sustancias causantes de doping y sustancias de uso terapéutico que pueden ser administradas a un caballo previo a un evento deportivo respetando los tiempos de eliminación del fármaco, siendo estas sustancias las que forman parte de la medicación controlada. Las aplicaciones más comunes de doping se conocen como doping positivo en el cual se altera el rendimiento del atleta de modo tal que este consiga ganar una competencia y doping negativo el alterar el rendimiento con el fin de que el atleta pierda o le sea imposible competir. Las técnicas más utilizadas para el control de doping se basan en la separación cromatográfica de las sustancias. Para ello los laboratorios acreditados en cada país cuentan con técnicas conocidas como cromatografía de gases y cromatografía líquida.

Palabras clave: dopping, rendimiento, equinos.

Abstract.

Doping is any method either physical or chemical that alters the performance and competitiveness of an equine. Over the years there have been many cultures who have used the horse as a working tool thus the need to increase animal performance data such ancient times that even the true beginning of this technique is unknown. Today the use of substances that alter the performance of the horse in a sporting event is strictly forbidden, even so, this prohibition is in the hands of the organizations involved in each country.

The International Equestrian Federation has established the existence of substances that should not be in the body of a horse at any time, called substances causing doping and substances for therapeutic use that can be administered prior to a sporting event horse respecting times Drug elimination, these substances are part of the controlled medication. The most common applications of doping are known as positive doping, in example alter the athlete's performance so that this manages to win a competition, and negative doping that is altering the performance to the athlete lost or impossible to compete. The techniques used to control doping are based on chromatographic separation of substances, for that accredited laboratories in each country have known techniques such as gas chromatography and liquid chromatography.

Keywords: dopping, performance, equines. 
Introducción.

En eventos deportivos donde el equino es el principal protagonista de estos mismos, es posible aumentar de manera artificial, ya sea el rendimiento físico como el instinto competitivo del animal. Esto involucra el uso de ciertas sustancias tales como: esteroides anabólicos y agentes estimulantes del sistema nervioso central. El término doping se define principalmente como el uso ilegítimo de drogas que aumenten o disminuyan el rendimiento físico del competidor y hoy las principales políticas anti doping en equinos se basan estrictamente en evitar el uso de estas sustancias. En los deportes donde es sometida la especie equina existe una gran demanda física, por ello en algunas ocasiones existen personas que actúan fuera de las normas deportivas establecidas, promoviendo el uso de drogas para alterar o recomponer el rendimiento físico del equino (Toutain, 2010).

Hoy en día la mayoría de los organismos reguladores deben distinguir el control de sustancias ilícitas y de sustancias terapéuticas, ya que el caballo puede recibir la administración de una sustancia ilícita que actúe interviniendo en su sistema nervioso central con intereses netamente particulares, o la administración de tratamientos con intereses legítimos que involucren el bienestar del animal. En ambos casos existen sustancias que ya sea para usos ilegales o terapéuticos están prohibidas dentro de muchos eventos deportivos.

En competencias deportivas del equino a menudo existe desgaste físico del animal donde el principal encargado de su bienestar es el Médico Veterinario. Muchas veces en estos eventos deportivos el rendimiento de los equinos se ve alterado debido a la administración ilegal de ciertas sustancias farmacológicas. El uso de drogas y medicamentos en equinos previo a una competencia deportiva es un problema preocupante ya sea para los jinetes, los veterinarios y el público amante de este tipo de eventos. Si bien existen medicamentos de los cuales se reconoce su uso terapéutico y el beneficio a la salud del equino, el uso de estos puede generar alteraciones en el rendimiento deportivo que este tenga en una competencia, por eso las organizaciones reguladoras son responsables de desarrollar reglas y controles antidoping para proteger el bienestar de los animales, de los jinetes y asegurar el interés del público cuando hay apuestas de por medio.

Esta revisión bibliográfica pretende informar de manera clara los aspectos más relevantes del doping en el equino de deporte, así como sus antecedentes históricos y la evolución de las distintas definiciones que han sido publicadas para el término doping, además esclarecer las distintas formas de doping que se practican en el deporte equino, describir las técnicas de control más utilizadas y evaluar aspectos importantes del doping a nivel nacional.

Historia de doping en el equino.

A lo largo del tiempo los seres humanos han utilizado sustancias para mejorar su rendimiento tanto en el combate como en la caza. Ya en el año 800 A.C los antiguos griegos tenían incorporado el deporte en su diario vivir el cual usaban como preparación de soldados para luchar. Durante el año 400 A.C el deporte en Grecia había alcanzado importancia y ya se realizaban grandes eventos masivos con gran cantidad de afluencia púbica donde los atletas eran vistos como una especie de héroe. Los honores, beneficios y recompensas otorgados a los atletas fue lo que llevó a la corrupción y a ese afán de querer superar su propio rendimiento (Higgins, 2006).

Según Cruz et al., (2009) la domesticación del caballo por parte del hombre data de tiempos inmemorables, donde su uso primordial se basaba en ser una herramienta de trabajo, medio de transporte o incluso formar parte de la guerra. Hoy en día el caballo también puede suponer un elemento de diversión ya que forma parte de eventos deportivos tales como, carreras, concursos de salto, polo y rodeo entre otros. Fue en ese entonces cuando el hombre intentó manipular y alterar las aptitudes físicas y el rendimiento del caballo mediante el uso de distintas hierbas y sustancias. Según Eurípides (480-406 a.C) el rey Diomedes de Tracia alimentaba a sus caballos con carne humana con el fin de hacerlos más salvajes e invencibles.

Existen indicios de que en la antigua Roma utilizaban una mezcla de agua con miel el que servía como estimulante para los caballos en las carreras. Hoy en día no se sabe de manera cierta si de verdad estos métodos eran o no eficaces (Higgins, 2006). Trioni (2008) describe que las primeras formas de doping 
resultaron ser muy violentas y proponían disminuir la resistencia física del contrincante más que tratar de mejorar la del propio equino. Por ello los caballos eran puestos bajo resguardo para así evitar estas acciones ilegítimas incluso para ese tiempo. Incluso el agua para el consumo del caballo era dispuesta en contendores herméticos donde se colocaban peces dentro con el fin de salvaguardar la integridad del agua. A principios del siglo XX había veterinarios que recomendaban administrar semillas de anís, miel y sandáraca, a fin de entregarle al caballo una fuente alternativa de fuerza y resistencia. Luego, un poco más tarde surgió una tendencia que consistía en administrar antes de un evento deportivo bebidas tales como, café, té, whisky, y otras bebidas alcohólicas con el mismo fin anteriormente mencionado.

En Norteamérica fueron pioneros en utilizar alcaloides de origen vegetal en el año 1890. Luego fueron introducidos a Inglaterra en el año 1896 y posteriormente se extendieron a Rusia, Francia, Alemania y Austria en el año 1901. Los entrenadores experimentaban con sustancias que presentaban una muy baja purificación y además desconocían las dosis adecuadas que debían administrar. Es por ello que se generaron muchos efectos nocivos debido al doping, se provocaron cambios negativos en el estado general del caballo resultando incluso en ocasiones con la muerte del animal antes o durante el evento deportivo, causando accidentes en las pistas que al mismo tiempo podían resultar en la muerte de los jinetes.

Hoy en día el Médico Veterinario tiene un rol muy importante en la prevención y control del doping, dándole importancia a dos aspectos, el primero netamente relacionado a un control deportivo y el segundo velar por el cuidado del paciente y que este no sea tratado con sustancias que puedan alterar su integridad y estado general (Cruz et al., 2009).

Definición de doping en el equino.

El origen exacto de la palabra "doping" muchas veces resulta confuso, ya que algunos autores aseveran que la palabra doping proviene de los cafres de África que utilizaban una sustancia tipo licor denominada "dop" y que utilizaban como estimulante, otros afirman que el término doping proviene de la palabra "dope" una expresión de origen americano refiriéndose al opio, una sustancia utilizada principalmente en caballos (Hol, 2009). Doping se define particularmente como la administración ilegal de cualquier agente o sustancia farmacológica en animales de competición con un fin específico, alterar el rendimiento físico del competidor para conseguir un deterioro de este mismo o bien conseguir aumentar sus capacidades físicas dentro de un evento deportivo (Rodríguez et al., 2004). Si hablamos de su etimología, el término doping proviene del sustantivo holandés "doop" el cual para este idioma significa mixtura o líquido empleado principalmente como excitante.

Si bien los intentos para alterar el rendimiento físico de los caballos, se remonta a hechos muy antiguos, fue recién en el año 1899 donde se incorporó por primera vez la palabra doping a un diccionario inglés. En este diccionario el término doping se define como una mezcla de opio y derivados de la morfina que se administraba a los caballos (Müller, 2010). En sus inicios la palabra doping se refería al uso de medicamentos con el fin de estimular caballos. Luego de los hipódromos el término comenzó a usarse en los estadios y hoy en día se define como doping a cualquier método que pueda alterar el rendimiento, perjudicar la salud del atleta, y atentar contra los valores del juego (De Rose, 2008).

El primer Congreso Internacional de Derecho del Deporte (1968), propuso como definición de dopaje: "La ingestión, por un individuo normal, por sí mismo o por inducción de otra persona, de algún producto o sustancia química que altere, modifique o restrinja directamente, o por sus efectos secundarios, la calidad de la participación de este individuo en una competición deportiva, independientemente de la vía por la que tal sustancia o producto se le haya administrado, así como también de la cantidad, contenido o preparación del producto, sin que se pueda alegar, como atenuante de la responsabilidad, el estar incapacitado temporalmente por lesión o enfermedad, ya que, en tales circunstancias, debe impedírsele competir". Fue la Agencia Mundial Antidopaje que en el 2007 publica el Código Mundial Antidopaje, quienes utilizan una definición más precisa relacionada netamente al incumplimiento de las normas establecidas (AMA) (2007). 
Aspectos legales.

En 1999 durante el primer Congreso Mundial antidopaje se crea la (AMA) con sede en Lausana, Suiza, luego 5 años más tarde en el segundo Congreso Mundial antidopaje realizado en Copenhague se aprobó el código mundial antidopaje. Este código contiene legislación específica para el control del dopaje y normas para llevar a cabo este control. Esta legislación internacional significó una gran lucha contra el doping, ya que promueve y recomienda sanciones aplicables a los atletas y a los responsables de realizar doping, luego estas sanciones se pueden establecer internamente por el estado y las federaciones deportivas de manera autónoma (Mussa, 2013).

En el año 2005 este código fue ratificado por la UNESCO en París, cuyo fin es promover la lucha contra el doping en cualquiera de sus formas. En el artículo 16 de este código se establece la regulación del doping en animales que participen en competencias deportivas, luego a partir de lo que dicta este artículo surgen las diversas normativas que establece la Federación Ecuestre Internacional (FEI) (Cruz et al., 2009). El deporte contribuye a mejorar la salud física y mental de las personas y además genera valores que son fundamentales dentro de la sociedad, tales como el trabajo en equipo, el respeto y la solidaridad. El deporte ocupa una posición privilegiada en nuestra sociedad actual, llegando a todas las edades y niveles socioeconómicos, ahora bien, la presión que genera la competitividad entre los deportistas conlleva a realizar acciones contrarias al espíritu deportivo. Internacionalmente las organizaciones han actuado con el fin de evitar el doping y así mantener vivo este espíritu deportivo, todos los riesgos que forman parte de esta práctica pueden ser minimizados a través de una regulación adecuada, imponiendo límites, deberes y responsabilidades al deportista y al equipo encargado (Mussa, 2013).

Dentro del doping está estrictamente prohibido tanto su uso, como su recomendación, al igual que su proposición y la facilitación de cualquier sustancia o método que forme parte de esta práctica. El transcurrir del tiempo y el avance constante de la farmacología han dado como fruto la aparición de nuevas técnicas que suelen usarse de forma ilegítima dentro del deporte por lo cual ha sido necesario que al mismo tiempo la legislación se vuelva más fuerte y dinámica (De Rose, 2008).

Organizaciones ecuestres involucradas en la aplicación de normativas.

1. Consejo Superior de la Hípica Nacional. Constituye el órgano supremo de la hípica nacional. Es un organismo asesor y dependiente del Ministerio de Hacienda, a quien corresponde regular, por medio de la aplicación del Código de carreras, la actividad hípica nacional.

2. Federación Ecuestre Internacional. Es la organización que gobierna internacionalmente los deportes ecuestres. Esta federación no rige a las carreras de caballos, rodeo ni al polo, que tienen federaciones independientes.

3. Federación Ecuestre de Chile. La Federación Ecuestre de Chile es el órgano rector de la actividad deportiva nacional; está afiliada a la Federación Ecuestre Internacional (FEI). Agrupa a ocho disciplinas: Adiestramiento, Concurso Completo de Equitación, Enduro Ecuestre, Enganche, Para ecuestre, Ponies, Rienda y Salto, además de seis Asociaciones (Santiago, Valparaíso, Concepción, Temuco, Sur y Enduro), quienes, a su vez, agrupan a más de 40 clubes en todo el país.

4. Federación Nacional de Rodeo. Es una institución de aficionados sin fines de lucro cuyo objetivo es regir y fomentar el rodeo en Chile y en sus relaciones con el extranjero.

5. Federación Chilena de Polo. Es el organismo oficial del deporte polo en Chile.

6. Agencia Mundial Antidopaje. La Agencia Mundial Antidopaje (WADA) es la organización internacional independiente creada en 1999 para promocionar, coordinar y monitorear la lucha contra el dopaje en el deporte en todas sus formas. 
Doping y medicación controlada.

Las sustancias ilícitas dentro del doping equino caben en dos categorías, en la primera categoría considerada como "doping" entran las sustancias prohibidas que no deben estar en el organismo del equino en ningún momento, luego dentro de la segunda categoría denominada "medicación controlada" entran las sustancias de uso terapéutico que tampoco deben estar presente dentro de una competencia. Las sustancias administradas ya sea ilegalmente o accidentalmente al organismo de un mamífero pueden afectar los siguientes sistemas:

Sistema nervioso

Sistema cardiovascular

Sistema respiratorio

Sistema digestivo

Sistema urinario

Sistema musculo-esquelético

Sistema circulatorio

Sistema endocrino

Sistema reproductivo

Sistema inmunológico (En este punto se pueden excluir las vacunas autorizadas para el control de ciertas enfermedades infecciosas) (Wong \& Wang, 2014). La (AMA) establece una clasificación para sus más de doscientas sustancias prohibidas, estas se clasifican en nueve categorías y tres métodos prohibidos.

1. Agentes anabolizantes

2. Hormonas peptídicas, factores de crecimiento

3. Agonistas Beta 2.

4. Antagonistas y moduladores de hormonas.

5. Diuréticos y otros agentes enmascarantes.

6. Estimulantes

7. Narcóticos

8. Canabinoides

9. Glucocorticoides

El control antidoping no solo se encarga de prohibir las infracciones respecto a la administración de medicamentos sino que al mismo tiempo protege el bienestar del caballo, dentro del código de medicación de la FEl las sustancias prohibidas dentro de cualquier evento deportivo se clasifican en medicamentos clase A y medicamentos clase B. Dentro de la clase A se encuentran sustancias que influyen en el rendimiento competitivo mediante una acción analgésica y alivio del dolor (AINE, anestésicos locales, etc) y dentro de la clase B se encuentran sustancias que pueden mejorar el rendimiento limitadamente (por ejemplo, los mucolíticos y supresores de la tos) o sustancias a los que el animal pudo haber sido expuesto accidentalmente (por ejemplo bufotenina, hordenina etc) (FEI, 2007).

La FEl establece que el uso de métodos terapéuticos que involucren el uso de fármacos cercano a un evento deportivo puede ser necesaria, pero aun así, resulta sustancialmente riesgoso el hecho de que al momento de realizar el control de doping el tiempo de eliminación del fármaco aún no ha transcurrido, debido a esto y para apoyar el trabajo del Médico Veterinario la FEI seleccionó una serie de medicamentos los cuales pueden ser utilizados en la práctica durante un tiempo previo al evento deportivo (FEI, 2007). 
Formas de doping en caballos de deporte.

\section{A.Doping positivo}

A1 Agudo: En este grupo se encuentran los estimulantes de corta duración como la cocaína, anfetaminas y estupefacientes, generalmente su administración es horas antes de un evento deportivo.

A2 Crónico: Para conseguir el efecto esperado es necesaria su administración durante un tiempo prolongado, es decir, repetir la dosificación durante semanas e incluso meses, en este grupo se encuentran los esteroides anabolizantes, vitaminas, y otros medicamentos que aumenten la movilización de glucógeno.

A3 Tranquilizantes: Están dentro de esta clasificación porque se utilizan principalmente en dosis pequeñas para conseguir tranquilizar o sedar a caballos nerviosos o demasiado excitables, para que así se vuelvan manejables y se favorezca su rendimiento en un evento competitivo.

A4 Trabajo interno: Se considera que la medicación para ganar es un proceso realizado por los responsables del caballo, ya que ellos son los principales interesados en obtener la victoria.

B. Doping negativo

B1 Tranquilizantes: Se administran grandes dosis de tranquilizantes ya que el propósito es disminuir el rendimiento del caballo.

B2 Trabajo externo: Naturalmente es un trabajo realizado por personal externo al grupo encargado del caballo y su propósito es disminuir el rendimiento del rival.

B3 Doping para restablecer el rendimiento normal

B4 Antiinflamatorios no esteroideales: Esta forma a menudo es permitida bajo reglas controladas de acuerdo a las distintas organizaciones involucradas. El principal AINE utilizado es la fenilbutazona.

B5 Antiinflamatorios esteroideales: Se utilizan los corticoides, generalmente son administrados mediante infiltraciones o intra articularmente para controlar el dolor local.

B6 Anestesia local: Se utilizan principalmente para insensibilizar alguna zona del cuerpo de manera selectiva y además como bloqueos nerviosos.

B7 Soluciones electrolíticas: Fluidos que a menudo están permitidos.

B8 Doping involuntario o accidental.

B9 Penicilina procaínica: Es un caso muy común que tras una terapia antibiótica se generen resultados positivos de procaína, la cual al liberarse dentro del organismo actúa como un potente estimulante, por otra parte es utilizada como anestésico local.

B10 Administración de cafeína y otras metilxantinas: vía oral mediante el consumo de alimentos contaminados.

B11 Positivos botánicos: Este punto hace referencia a la existencia de contenidos vegetales que poseen características los cuales pueden confundirse con algunos principios farmacológicos. Hoy en día las técnicas modernas de análisis evitan que se produzcan estos falsos positivos.

B12 Medicación para enmascarar el uso de otras sustancias Utilizadas generalmente para acelerar o disminuir la excreción de la orina. Alcalinización de la orina para retrasar la excreción de sustancias como las anfetaminas. Administración de dipirona o polietilenglicol.

B13 Medicación para diluir otras sustancias

B14 Diuréticos: El uso de furosemida u otros diuréticos como ácido etacrínico e hidroclorotiazida están implicados en el aumento del volumen de orina lo que permite disminuir las concentraciones de sustancias ilícitas.

C. Otras formas de doping.

C1 Doping sanguíneo: Consiste en la extracción de cierta cantidad de sangre del caballo para el posterior almacenaje de eritrocitos, luego días antes de la competencia se vuelve a administrar la sangre extraída, esto con el fin de que el caballo corra con una proporción más elevada de eritrocitos por lo tanto 
aumente los niveles de oxígeno en la sangre. La efectividad de este procedimiento no ha sido del todo demostrada (Tobin, 1989).

Sustancias permitidas.

Dentro de las reglas y regulaciones de la Federación Ecuestre Internacional existen ciertas sustancias las cuales su uso está permitido. Estas sustancias son: vitaminas B, aminoácidos y electrolitos, la vía de administración generalmente es la vía oral, aunque está en la decisión del veterinario a cargo preferir la administración intravenosa o intramuscular. También en algunos casos están permitidas las terapias preventivas como por ejemplo el uso de condroitina y glucosamina. Sin embargo, el uso de sustancias desconocidas como hierbas medicinales y tónicos los cuales no presentan ingredientes detallados y su formulación química es desconocida están prohibidas previo a una competencia, ya que los productos herbales pueden actuar como sedante o energizante y por ende afectar el rendimiento del caballo en una carrera (FEI, 2014).

Metabolismo de las drogas.

Gran parte de los fármacos y drogas causantes de doping se convierten en metabolitos para facilitar su excreción a través de la orina. Existen dos fases por las cuales el fármaco pasa para transformarse en metabolitos estas son, metabolismo de fase I y II. El metabolismo de fase I consta de reacciones tales como la oxidación, reducción e hidroxilación todas ellas catalizadas por el citocromo ubicado principalmente en el hígado. Por otra parte, el metabolismo de fase II consta de reacciones tales como la glucoronidación y sulfatación y estas ocurren en el cuerpo (Badoud, et al, 2011). Estas fases son denominadas procesos de biotransformación ya que la sustancia transforma su estructura química mediante reacciones enzimáticas, el resultado de este proceso culmina en el aumento de la hidrofilidad de las drogas y la disminución de su toxicidad. Por otra parte, estas reacciones de biotransformación pueden provocar la bioactivación de fármacos en cuyo caso el metabolito es más toxico y/o más activo que el fármaco original (Wissenbach, 2012).

Control de doping.

El control de doping puede ser mediante muestras como la orina, la sangre, o ambos (De Rose, 2008). Hoy en día el control de doping en el deporte equino es muy diferente al control que se realiza en eventos deportivos donde el atleta es el humano ya que en este último el doping se realiza con un solo fin el cual es principalmente mejorar el rendimiento físico. Por otra parte, en el deporte equino se utilizan sustancias ya sea con la intención de mejorar el rendimiento o de disminuirlo, pudiendo alterar de manera fraudulenta el resultado del evento deportivo (Wong \& Wan, 2014). Tomando en cuenta las pruebas para el control de doping en el deporte equino, las muestras de sangre ofrecen una cierta ventaja respecto a las muestras de orina ya que la sangre puede ser extraída en cualquier momento y en cantidades requeridas para realizar los respectivos análisis. En la mayoría de los casos la sangre ha resultado ser la única muestra disponible ya que obtener una muestra de orina puede ser más complicado (Kwok et al. 2010). Los análisis de sangre son muy efectivos a la hora de encontrar la droga aun sin metabolizar, mientras que para la detección de metabolitos es estrictamente necesaria la muestra de orina ya que muchos medicamentos son altamente metabolizados (Kwok et al., (2010).

Tipos de análisis.

La función principal de los laboratorios enfocados en control de doping es detectar analitos con distintas propiedades físico-químicas, diferentes rangos de polaridad, distintos niveles de $\mathrm{pH}$, además de diferencias en tamaño molecular y propiedades farmacocinéticas (Kioussi et al, 2013). Existe un gran número de estructuras químicas que forman parte de las sustancias usadas como doping y para confirmar su 
presencia en el organismo es necesario el uso de ciertas estrategias analíticas (Badoud et al., 2011). Para la detección de una gran cantidad de sustancias ilegales existen numerosos métodos de análisis. Estos métodos se fundamentan en la separación cromatográfica de la sustancia: por una parte existe la cromatografía de gases (CG) y la cromatografía líquida (CL), seguido inmediatamente por la espectrometría de masas (EM) (Ludwig et al , 2014). Los métodos cromatográficos han resultado ser uno de los estudios más importantes respecto al análisis de ciertas sustancias. Tanto la cromatografía de gases como la cromatografía de líquidos tienen amplias áreas de aplicación en laboratorios de todo el mundo (Kaal \& Janssen, 2007).

Las pruebas utilizadas para detección de uso de drogas permiten evidenciar la presencia de metabolitos en la orina que indiquen el uso de sustancias a pesar de que estas pueden no ser detectadas (Kioussi, 2013). El avance de los estudios farmacológicos ha sido muy importante respecto al aumento de la sensibilidad de detección de ciertas sustancias que antes eran indetectables, pero al mismo tiempo han progresado las ciencias biológicas que se han encargado de desarrollar más sustancias prohibidas, sin embargo, no importa la progresión de los métodos de detección, ya que la brecha es amplia y la aparición de nuevas sustancias prohibidas es inevitable (Wong \& Wan, 2014).

Fundamentos teóricos de la cromatografía de gases y cromatografía líquida.

La cromatografía de gases es una técnica analítica que se encarga de separar mezclas de sustancias en componentes individuales, se utiliza para identificar ciertos analitos y medir sus concentraciones. Se fundamenta en vaporizar una mezcla con la ayuda de un gas inerte (fase móvil) y traspasar cada compuesto de la mezcla a través de una columna cromatográfica (fase estacionaria). Los componentes son separados al atravesar la fase estacionaria debido a que tienen distintas tasas de migración, y es aquí donde ocurre la separación de cada compuesto que forme parte de la sustancia (Haneef et al, 2013).

La capacidad de tener una migración diferencial entre cada compuesto de una mezcla da como resultado la selectividad cromatográfica, debido a todo esto, es necesario que los compuestos analizados tengan una suficiente volatilidad ya que sustancias que posean moléculas demasiado grandes no podrán pasar a la fase móvil por ende no podrán ser analizadas con éxito (Kaal \& Janssen, 2007). Por otra parte la cromatografía líquida es una técnica analítica en donde la sustancia es inyectada en una fase móvil líquida la cual se hace pasar por una fase estacionaria siendo aquí donde los componentes de una muestra se distribuyen de manera distinta logrando desplazarse en bandas discriminadas y cada una de las sustancias introducidas en el sistema traspasará la fase estacionaria con un tiempo diferente, es decir, se estarán separando (Kioussi et al, 2013).

Toma de muestra.

Es importante considerar que el proceso de toma de muestra consta de aspectos legales, en los que se debe llevar un seguimiento estricto de la muestra a través de cadenas de custodia, con documentación que indique el camino que ha recorrido la muestra hasta llegar al laboratorio.

El proceso de control de doping comienza inmediatamente con la toma de muestra donde el recipiente contenedor de la muestra debe ser completamente estéril y sellado para evitar cualquier tipo de contaminación indeseada. Luego de obtener la muestra, ésta debe ser sellada herméticamente asegurando su integridad durante el transporte hasta el laboratorio.

El principal fluido corporal utilizado para realizar control de doping es la orina. Esta muestra es relativamente fácil de obtener y además es mucho más práctico al momento de realizar los análisis ya que es posible encontrar concentraciones más altas de la sustancia y sus metabolitos, respecto a lo que se puede encontrar en la sangre.

La toma de muestra se realiza inmediatamente luego de un evento deportivo donde los equinos seleccionados son dispuestos en instalaciones seguras en las cuales se procede a recoger la muestra de orina y sangre respectivamente.

Momento de la toma de muestra de sangre. 
De la muestra principal debe obtenerse una segunda muestra la cual corresponde a una pequeña porción que será vertida en un contenedor separado. Esta muestra secundaria se almacenará en un sitio seguro bajo condiciones de congelamiento, separado físicamente de la muestra principal las cuales están destinadas a los respectivos análisis de laboratorio. En eventos deportivos, si el laboratorio entrega informes de doping positivo, es decir, si evidencia la presencia de sustancias prohibidas o sobredosis de fármacos autorizados, el propietario o entrenador del caballo tienen la opción de un segundo análisis con la contra muestra que anteriormente se obtuvo, por ende, en un segundo laboratorio autorizado se repite el análisis para confirmar la presencia de drogas en el caballo. Si en los análisis realizados en el segundo laboratorio no se confirma la presencia de sustancias ilícitas o excedentes de otras sustancias autorizadas, no se tomarán medidas reglamentarias (Baker \& Stanley, 2004).

Tiempo de detección.

El tiempo de detección corresponde al periodo de tiempo durante el cual una sustancia o sus metabolitos permanecen en el organismo de un caballo y es posible que esta pueda ser detectada. Los laboratorios proporcionan el listado con las sustancias y sus tiempos de detección solamente como una guía, posteriormente es el Médico Veterinario el que debe encargarse mediante decisiones profesionales la administración de medicamentos tomando en cuenta los tiempos de detección (FEI, 2014).

Respecto al tiempo en el que una sustancia puede ser detectada posterior a una toma de muestra está afectado por varios factores entre los que se encuentran el tipo de terapia (fármaco, formulación, vía de administración, dosis y frecuencia de administración), el tipo de muestra (orina, sangre, o pelo) y la sensibilidad del método analítico empleado (Wong \& Wan, 2014). Además, existe una variabilidad individual entres los caballos en relación a la farmacocinética de las drogas y la disposición de la droga en el plasma y la orina. Esta variabilidad se debe a factores tales como la raza, edad, sexo y peso (Toutain, 2010). Los tiempos de detección publicados proporcionan una información fidedigna y clara, aun así, existen limitantes ya que los estudios farmacocinéticos para evaluar los tiempos de detección de una sustancia han sido realizados en laboratorios bajo condiciones de reposo. Por otra parte, en ejercicio y programas deportivos la influencia sobre la eliminación de las drogas es completamente distinta a la influencia generada bajo condiciones experimentales de laboratorio. Estos planteamientos resultan ser teóricos ya que la distribución y comportamiento de las sustancias en organismos biológicos resulta ser una ciencia no tan exacta.

En muchos países existen políticas de tolerancia cero respecto al doping, esto ha generado la aplicación de sanciones estrictas incluso ante la presencia de sustancias que no han tenido ninguna intención de alterar el rendimiento, por otro lado, hay países en los que las autoridades hípicas permiten competir a los caballos bajo el uso de ciertas sustancias como la fenilbutazona y la furosemida, pero se debe hacer público que el caballo compite bajo el efecto de dicha sustancia.

La Federación Ecuestre Internacional (FEI) junto con el Comité Científico Europeo de Carreras de Caballos (EHSLC) están determinando los tiempos de detección para un gran número de sustancias que puedan ser usadas con fines terapéuticos. Estos tiempos de detección se hacen solo para sustancias de uso terapéutico, como es el caso de analgésicos, corticoides o diuréticos. En cambio, para el otro grupo de sustancias su uso está absolutamente prohibido por lo tanto su presencia en los análisis se considera una infracción, en este grupo se encuentran los esteroides anabolizantes y las anfetaminas (Rodríguez, 2012).

\section{Situación en Chile.}

El Médico Veterinario tiene un papel fundamental en la entrega de atención médica a los caballos en todo momento, por lo mismo está en sus manos velar por el bienestar de los atletas otorgando tratamientos frente a cualquier afección, y es responsable frente al propietario del caballo ya que éste el que tiene especial interés en mantener al atleta compitiendo en óptimas condiciones (Campbell, 2013).

Antes de administrar cualquier sustancia a un caballo de competencia, el personal encargado y el veterinario deben tener conocimiento y comprobar si la sustancia se encuentra prohibida bajo los estatutos de la organización nacional responsable, es por ello, que debe existir un asesoramiento adecuado para 
retirar al caballo antes del evento deportivo si es que aún el tiempo de eliminación del fármaco no ha transcurrido (FEl, 2014).

Respecto a las pruebas que se realizan a nivel nacional dentro de la hípica, existe una disposición propia para realizar el control de doping. Cada país posee una organización propia, aunque de igual forma debe respetar las normas provenientes de la FEI (Rodríguez, 2004).

En Chile el único laboratorio que realiza análisis y control antidoping es el laboratorio de la Facultad de Química y Farmacia de la Universidad de Chile. Este laboratorio trabaja con muestras de diferentes atletas.

\section{Código de Carreras}

Según el artículo 255 de las drogas y otras substancias perteneciente al código de carreras se prohíbe suministrar medicamentos, drogas u otras sustancias químicas o cualquier agente físico, a excepción de furosemida, a los caballos inscritos para participar en carreras públicas. Además, se establece que los medicamentos, drogas u otras sustancias químicas se dividirán en las siguientes clases:

Clase I: Son aquellos estimulantes y drogas depresoras que tienen el más alto potencial para alterar la performance de carrera, y no poseen un uso médico específico en el caballo de carrera.

Clase II: Son aquellos estimulantes o drogas con un alto potencial para afectar la performance. No son agentes terapéuticos aceptados en los caballos de carrera. Se encuentran incluidas en esta Clase aquellas sustancias terapéuticas con altas posibilidades de uso indebido.

Clase III: Son aquellas drogas y sustancias que pueden o no tener un uso médico aceptado en el caballo de carrera, pero cuyo efecto farmacológico sugiere una capacidad menos para afectar la performance que aquellas pertenecientes a la Clase II.

Clase IV: Son aquellas medicamentaciones terapéuticas que tienen menos capacidad para afectar el rendimiento de un caballo.

Clase V: Son aquellas medicamentaciones terapéuticas para las cuales se han establecido umbrales o límites permitidos. Por otra parte, dentro de las normas generales establecidas por la federación del rodeo chileno existen ciertas sustancias permitidas en esta competición:

Antibióticos

Enrofloxacino.

Penicilina Sódica.

Gentamicina.

Estreptomicina.

Sulfas.

Trimetoprina.

Neomicina.

Reconstituyentes.

Vitaminas.

Minerales.

Antiinflamatorios.

Fenilbutazona (Oral y endovenosa).

Flunixin.

Meglumine.

Tranquilizantes.

Acepromacina.

Diuréticos.

Furosemida.

Vasodilatadores.

Isoxsuprine.

Antidiarreicos.

Caolin.

Pectina.

Coleréticos y colagogos. 
Membutone.

\section{Discusión.}

Uno de los factores más importantes respecto al doping en equinos de deporte es sin duda el abuso de medicamentos los cuales generalmente pueden ser o no de uso terapéutico. Como se ha mencionado anteriormente existen drogas que modifican el metabolismo de un equino en una competencia de tal forma que alteran el resultado de una competición y por otra parte, existen sustancias terapéuticas que no alteran el rendimiento, pero aun así su presencia o la presencia de sus metabolitos en muestras de orina y sangre ésta estrictamente prohibido según las distintas jurisdicciones de cada país.

Existen medicamentos que son administrados a equinos de deporte solamente con finalidad curativa (Toutatin, 2010), por otra parte, según (De Rose, 2008) indica que las distintas normativas establecidas en algunos países no disponen de diferencias entre las drogas y consideran causante de doping a cualquier sustancia sea cual sea su finalidad.

Según Toutain (2010), en la actualidad la mayoría de los controles se realizan utilizando orina, por otra parte, Wong \& Wang (2014) afirman que dentro de los estudios deberían considerar el uso de la sangre ya que el uso de plasma como matriz para el control de la medicación es más eficaz en relación a la farmacocinética del medicamento. Este tipo de muestra es importante, ya que las concentraciones plasmáticas del medicamento sin metabolizarse son más altas. Otros autores como Dunnett \& Lees (2003) establecen que el pelo de un equino resulta ser una matriz importante sobre todo para la investigación y evaluar un historial frente a la exposición de ciertas drogas como los esteroides anabólicos, aunque la limitación de esta muestra es que es de fácil contaminación. Por otra parte, en esta revisión bibliográfica se mencionó que existen fármacos altamente metabolizables por ende su único estudio y control es más eficaz llevarlo a cabo mediante muestras de orina (Kwok et al., 2010).

De acuerdo a los aspectos descritos en la presente revisión bibliográfica, el doping ha resultado ser una práctica totalmente contraria a la ética y el espíritu de las competencias que involucran a la especie equina siendo una costumbre peligrosa que pone en riesgo la salud y el bienestar tanto del animal como del atleta humano. Respecto al análisis histórico el doping se ha llevado a cabo durante muchos años tanto en el deporte como en otras actividades donde el caballo ha sido dispuesto al servicio del hombre, y hoy en día no existen señales de que este actuar fraudulento comience a disminuir.

El Médico Veterinario tiene un papel muy importante ante la asistencia y atención médica a los caballos durante y entre cada una de las competencias en que estos participen y la relación entre médico y paciente debe ser intima, pero aun así existe la intervención de terceros que denigran el espíritu del deporte con un actuar antiético.

Hoy en día las sanciones incluidas dentro de las legislaciones nacional e internacionalmente son un signo importante de que en el deporte existe responsabilidad ética que promueve la protección de los atletas y del público involucrado en dichos eventos deportivos. Es por esto que todo control antidoping representa un amparo al espíritu deportivo y es la clave para disminuir el afán de corromper los valores que presenta el deporte equino. Respecto al uso de drogas causantes de doping se establece que toda responsabilidad recae sobre los veterinarios, los entrenadores, árbitros, propietarios, oficiales y sobre todo, las federaciones nacionales de cada país. Todos ellos son responsables de cooperar para detener el doping, educar a la población y establecer vigilancia en las competiciones. 
Sustainability, Agri, Food and Environmental Research 3(4), 2015: 1-13

ISSN: 0719-3726

Bibliografía.

Antidoping \& Medication control. http://www.britishhorseracing.com/resources/equine-science-andwelfare/medicationand-doping-faqs.asp (visited 20 th may 2014)

Authie E., Garcia P., Popot M., Tountain P., Doucet M. 2010. Effect of an endurancelike exercise on the disposition anddetection time of phenylbutazone and dexamethasone in the horse: Application to medication control. Equine Veterinary Journal, 42: 240- 247.

Badoud F., Guillarme D., Boccard J., Grata E., Saugy M., Rudaz S., Veuthey J. 2011. Analytical aspects in doping control: Challenges and perspectives. Forensic Science International, 213: 49-61.

Baker C., Stanley S.2004. Detection of drug use in performance horses. Equine Sports Medicine and Surgery, 65: $1253-1266$.

Campbell M.2013. The role of veterinarians in equestrian sport: A comparative review of ethical issues surrounding human and equine sports medicine. The Veterinary Journal, 197: 535-540.

Cruz L., Gálvez., Pardo G., Encinas M. 2009. Dopaje en veterinaria II: Caballos y perros. Revista Complutense de Ciencias Veterinarias, 3: 227-234.

Deventer K., Pozo O., Verstraete A., Van Eenoo P. 2004. Dilute-and-shoot-liquid chromatography-mass spectrometry for urine analysis in doping control and analytical toxicology. Trends in Analytical Chemistry, 55:1-13.

De Rose E. 2008.Doping in Athletes - An Update. Clinics in sports medicine 27: 107 - 130.

Dunnet M., Lees P. 2003. Trace element, toxin and drug elimination in hair with particular reference to the horse. Research Veterinary Sciences 75: 89-101.

FEI .2007 Equine anti-doping decisions. https://www.fei.org/fei/your-role/athlete/fei-tribunal/2007decisions (visited 20th may 2014)

FEI (2014) Prohibited Substances List . http://www.fei.org/fei/cleansport/ad-h/prohibited-list (visited 15 th june may 2014)

Guan F., Uboh C., Soma L., Luo Y., Rudy J., Tobin T. 2005 Detection, quantification and confirmation of anabolic steroids in equine plasma by liquid chromatography and tandem mass spectrometry. Journal of Chromatography B, 829:56-68.

Haneef J., Shaharyar M., Husain A., Rashid M., Mishra R., Parveen S., Ahmed S., Ahmed N., Kumar D. 2013 ApplicationofLC-MS/MS forquantitative analysis of glucocorticoids and stimulants in biological fluids. Journal of Pharmaceutical Analysis, 5: 341-348.

Hinchcliff K. 2004 Drug effects on performance. Equine Sports Medicine and Surgery, 66: 1268 - 1286.

Higgins A. 2006 From ancient Greece to modern Athens: 3000 years of doping in competition horses. Blackwell Publishing Ltd, 29: 1-10.

Ho E., Leung D., Leung G., Wan T., Wong H., Xuc X., Yeung J. 2007 Metabolic studies of mesterolone in horses. Analytica Chimica, 596: 149-155.

Ho E., Leung D., Wan T., Yu N., 2005 Metabolic studies of methenolone acetate in horses. Analytica Chimica, 540: 111-119.

Holt R., Mulligan I., Sönksen P. 2009 The history of doping and growth hormone abuse in sport. Growth Hormone \& IGF Research, 19: 320-326.

Kaal E., Janssen H.2007 Extending the molecular application range of gas chromatography. Journal of Chromatography A, 1184: $43-60$.

Kioussi M., Lyrisa E., Angelis Y., Tsivou M., Kouooaris M., Georgakopoulus C., 2013 A generic screening methodology for horse doping control by LC-TOF-MS, GCHRMS and GC-MS. Journal of Chromatography B., 941, 69-80.

Kwok W., Leung D., Leung G., Wan T., Wong C., Wong J., 2010 Screening of drugs in equine plasma using automated on-line solid-phase extraction coupled with liquid chromatography-tandem mass spectrometry. Journal of Chromatography A, 1217: 3289-3296.

Ludwig S., Ginkel L., Nielen M. 2014 Screening of protein biomarkers for sports doping and veterinary control screening of protein biomarkers for sports doping and veterinary control. Trends in Analytical Chemistry, 57: 47-63. 
Mussa F.2013 Anti-doping control and public health: limits to the exposure of human health to risk in the name of sporting glory. Revista de Saúde Pública: 47.

Müller R. 2010 History of doping and doping control. Doping in Sports: Biochemical Principles, Effects and Analysis Handbook of Experimental Pharmacology. 195(195):1-23.

Pagan J., Waldriage B., Whitehouse., Dalglish., Fuchs S., Goff M.2013 Furosemide reduces the energetic cost of exercise in Thoroughbreds independent of its effect on EIPH. Journal of Equine Veterinary Science, 33: 321-399.

Petrone N. 2011 Acción de la Furosemida en la Hemorragia Pulmonar Inducida por el Ejercicio como, Elemento Químico de Doping. Tesis de Magister, Universidad de Buenos Aires 55 pp.

Pichini S. 2009 Growth hormone in sport: beyond Beijing 2008. Ther Drug Monit. Reglamento control doping de la federación del rodeo chileno. 31: 3-13.

Rodríguez M., Fragío C., Jüttner C., González M. El doping en caballos de competición. http://www.redalyc.org/articulo.oa?id=55919726007_visited 15 th August 2014)

Segura J., Gutiérrez G., Ventura., Pascual J., Bosch., Such., Nikolovski Z., Pinyot A. 2008.Growth hormone in sport: beyond Beijing. 31(1):3-13

Tobin T. Drugs and the performance horse. Equine Veterinary Journal 14: 298.

Tobin T. 1989 Immunoassay detection of drugs in racing horses. XI. ELISA and RIA detection of fentanyl, alfentanil, sufentanil and carfentanil in equine blood and urine. Research Communications in Chemical Pathology and Pharmacology 63: 129-52.

Tobin T., Harkins J., Sams R. 1999 Testing for therapeutic medications: Analytical/ pharmacological relationships and the need for "limitations" on the sensitivity of testing for certain agents. Journal of Veterinary Pharmacology and Therapy 22: 220-233.

Toutain P.2010 Veterinary Medicines and Competition Animals: The Question of Medication versus Doping Control. Comparative and Veterinary Pharmacology Handbook of Experimental Pharmacology, 199: $315-339$.

Trioni A. 2008 El doping en el equino deportivo http://www.produccionanimal.com.ar/produccion equinos/produccion equina en general/72-doping.pdf (visited 20th may 2014)

Watson M. 2011 Furosemide. Journal of Exotic Pet Medicine, 20: 60-63.

Wissenbach D.2012 Development of the First Metabolite-based LC-MS Urine Drug Screening Procedure. Toxichem Krimtech, 88: 88-92.

Wong J., Wan T.2014 Doping control analyses in horseracing: A clinician's guide. The Veterinary Journal 200: $8-16$. 